\title{
Identification of somatic copy number variations in plasma cell free DNA correlating with intrinsic resistances to EGFR targeted therapy in T790M negative non-small cell lung cancer
}

\author{
Lucheng Zhu ${ }^{1,2,3 \#}$, Jiafeng Liang ${ }^{1,2 \#}$, Bing Xia ${ }^{3}$, Yasi Xu ${ }^{1,4}$, Ziliang Qian ${ }^{5}$, Shenglin Ma ${ }^{1,2,6}$, Shirong Zhang ${ }^{1,4,6}$ \\ ${ }^{1}$ Center for Translational Medicine, Key Laboratory of Clinical Cancer Pharmacology and Toxicology Research of Zhejiang Province, The Affiliated \\ Hangzhou Hospital of Nanjing Medical University, Hangzhou 310006, China; ${ }^{2}$ Department of Oncology, The Affiliated Hangzhou Hospital \\ of Nanjing Medical University, Hangzhou 310006, China; ${ }^{3}$ Department of Oncology, Hangzhou Cancer Hospital, Hangzhou 310002, China; \\ ${ }^{4}$ Center for Translational Medicine, Key Laboratory of Clinical Cancer Pharmacology and Toxicology Research of Zhejiang Province, Affiliated \\ Hangzhou First People's Hospital, Zhejiang University School of Medicine, Hangzhou 310006, China; ${ }^{5}$ Prophet Genomics Inc, San Jose, CA, USA; \\ ${ }^{6}$ Department of Oncology, Affiliated Hangzhou First People's Hospital, Zhejiang University School of Medicine, Hangzhou 310006, China \\ Contributions: (I) Conception and design: S Zhang, S Ma; (II) Administrative support: S Zhang, S Ma; (III) Provision of study materials or patients: \\ B Xia; (IV) Collection and assembly of data: J Liang, B Xia, Y Xu; (V) Data analysis and interpretation: L Zhu, Z Qian, S Zhang; (VI) Manuscript \\ writing: All authors; (VII) Final approval of manuscript: All authors. \\ \#These authors contributed equally to this work. \\ Correspondence to: Shirong Zhang. Affiliated Hangzhou First People’s Hospital, Nanjing Medical University, No. 6 Xiaonv Road, Shangcheng District, \\ Hangzhou 310006, China. Email: shirleyz4444@163.com; Shenglin Ma. Affiliated Hangzhou First People’s Hospital, Nanjing Medical University, \\ No. 261 Huansha Road, Shangcheng District, Hangzhou 310006, China. Email: mashenglin@medmail.com.cn.
}

Background: About 20-30\% EGFR-mutant non-small lung cancer show intrinsic resistance to EGFR targeted therapies. Compared to T790M positive in acquired resistance patients, little is known about EGFR-TKI intrinsic resistance for T790M negative patients.

Methods: Thirty-one patients with advanced stage lung cancer, including 18 patients with intrinsic resistance ( $\mathrm{PFS}<6$ months) and 13 patients with acquired resistance ( $\mathrm{PFS}>36$ months) but are negative for plasma T790M were recruited in the study. Plasma cell free DNA was profiled by low coverage whole genome sequencing with median genome coverage of $1.86 \mathrm{X}$ by Illumina X10. Sequencing coverage across chromosomes was summarized by samtools, and normalized by segmentation analysis as provided by $\mathrm{R}$ package 'DNACopy'.

Results: The most frequent chromosomal changes were found on chr7, chr1 and chr8. Among them, chr7p gains were found in 12 (66.7\%) intrinsic resistance and 4 (30.7\%) acquired resistance patients. The gene EGFR was found located on the focal amplification peak of chr7p. The performance of $7 \mathrm{p}$ gain to predict intrinsic resistance reaches AUC $=0.902$. Similarly, focal amplifications were also found on chromosome 5,

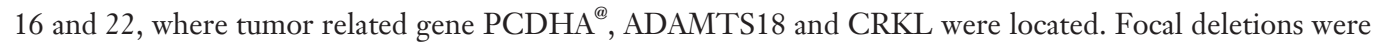
also found in chr1, 8, 10 and 16, where genes SFTPA1/2, DLC1, PTEN and CDH1 are located.

Conclusions: The results suggest cell free DNA copy number might be a useful peripheral blood tumor biomarker for predicting intrinsic resistance of EGFR targeted therapy and prognosis.

Keywords: Copy number variations (CNV); plasma cell free DNA (cfDNA); intrinsic resistances; T790M negative; non-small cell lung cancer (NSCLC)

Submitted Sep 04, 2019. Accepted for publication Nov 29, 2019.

doi: $10.21037 /$ jtd.2019.12.97

View this article at: http://dx.doi.org/10.21037/jtd.2019.12.97

(c) Journal of Thoracic Disease. All rights reserved. 


\section{Introduction}

Lung cancer is the leading cause of cancer death worldwide. In the past decades, significant improvements have been made for lung cancer treatment, based on discoveries of molecular biomarkers and disease progression mechanisms. A landmark of these improvements is epidermal growth factor receptor (EGFR) targeting therapy for patients with advanced lung cancer bearing EGFR activating mutations $(1,2)$. However, clinical outcomes for lung cancer still remains unsatisfactory, with 5 -year survival rate of less than $20 \%(3)$.

Clinical studies have revealed that lung cancer with activating EGFR mutations that are initially responsive to EGFR-tyrosine kinase inhibitors (EGFR-TKIs) will develop acquired resistance to TKI after a median progression-free survival (PFS) of 10-16 months $(2,4)$. On the other hand, $20-30 \%$ of patients with non-small cell lung cancer (NSCLC) harboring activating EGFR mutation show no objective tumor regression to initial EGFR-TKI treatment, and this subgroup of NSCLC has been defined as with intrinsic or primary resistance to EGFR-TKIs.

Studies have revealed that the secondary T790M mutation of EGFR is the major cause of the acquired EGFR-TKI resistance in lung cancer (5). The thirdgeneration of EGFR-TKI, aiming targeting the acquired EGFR T790M resistance mutation, has thus been developed. Clinical studies showed that osimertinib, a first third-generation of EGFR-TKI received FDA and EMA approval, demonstrated significant clinical effects with $70 \%$ objective response rate and 10 months progression free survival in metastatic EGFR-mutant NSCLC patients failure to first-generation EGFR-TKIs (6). Other causes for the acquired EGFR-TKI resistance in lung cancer include c-MET amplification (7), HER2 and PIK3CA mutation (8).

Very little is known about intrinsic resistance of EGFRTKI, however, especially for the lung cancers that are with activating EGFR mutation but negative for T790M. Of note, most studies focused on single gene alteration, but it is needed to be indicated that multiple resistance mechanisms may co-exist because of tumor heterogeneity $(9,10)$. Therefore, novel perspective study for EGFR-TKI resistance is needed.

Genomic instability involves a transient phase of tetraploidization. Tetraploid cells can undergo asymmetric cell division or chromosome loss, leading to increased tumor heterogeneity and multidrug resistance (11).
Experimental evidence further revealed that chromosomal instability enables tumor adaptation with aneuploid fitness landscape $(12,13)$. In lung cancer cells, chromosome 7 aneuploidy was found to be one of the most important events for cancer development. These deregulations or variations of Chromosome 7 can be frequently detected in malignant lung cancer and pre-cancerous lesions cells, or even in lung bronchial cells, but not in health lung tissues cells (14-16). Chromosome aneuploidy detection has been used for prenatal tests through plasma cell free DNA, with minimal false positives and false negatives (17). Similar to fetal tissues and cells, tumors also keep shedding DNA into peripheral blood stream. As such, technology of ctDNA (circulated tumor DNA) has been successfully applied in clinic to detect biomarkers or cancer somatic mutations such as EGFR mutations for predicting potential benefits of targeted therapies. In addition, detection of chromosomal copy number changes with ctDNA technology has also been reported in patients with breast cancer (18), hepatocellular carcinoma (19) and lung cancer (20).

The criteria for clinical defining intrinsic EGFRTKI resistance for lung cancer have not been established. Jackman et al. (21) proposed criteria for acquired resistance of EGFR-TKI in lung cancer patients with mutant EGFR, including that patients achieve a partial or complete response or develop a stable disease in response to EGFRTKI monotherapy ( $>6$ months). Of note, patients with PFS less than 6 months were recruited in the study, and thus the lung cancer of these patients potentially harbored intrinsic resistant to EGFR-TKI treatment.

Low pass whole genome sequencing approach with an optimized bioinformatics pipeline, ultra-sensitive chromosomal aneuploidy detector (UCAD), were used to screen chromosomal aneuploidy, especially chr7 aneuploidy, by using plasma cell free DNA in EGFR targeted therapy intrinsic resistant patients.

\section{Methods}

\section{Patients}

Thirty-one lung cancer patients and ten health volunteers were enrolled in this study. All lung cancer patients relapsed after EGFR-TKI treatment. The protocol of the study was approved by the Institutional Review Board of Hangzhou First People's Hospital (No. HZFH CA15-02). All recruited patients and health volunteers have signed a written 
informed consent.

\section{Sample collection and DNA extraction}

Blood samples were collected within 14 days after the development of TKI resistance as assessed by the physician according to the Jackman criteria (21) and before the start of the following treatment. Approximately $10-15 \mathrm{~mL}$ of peripheral blood was collected in a cell-free DNA protection vacuum tube (AmoyDx, Xiamen, Fujian, China), which contains a cell-free DNA protection reagent to keep DNA stable for 7 days at $4-25^{\circ} \mathrm{C}$. Blood samples were transported to the Center for Translational Medicine of Hangzhou First People's Hospital within 36 hours for further processing. For DNA extraction, the blood samples were centrifuged at $2,500 \times \mathrm{g}$ for 10 minutes at $4{ }^{\circ} \mathrm{C}$, and the supernatant was transferred to a new tube for further centrifugation at $15,800 \times \mathrm{g}$ for 15 minutes at $4{ }^{\circ} \mathrm{C}$. The collected plasma supernatant was then stored at $-80{ }^{\circ} \mathrm{C}$. Cell-free DNA from $1.5 \mathrm{~mL}$ plasma was extracted with a QIAamp Circulating Nucleic Acid kit according to the manufacturer's instructions (Qiagen, Hilden, Germany).

\section{ARMS assays for testing EGFR T790M mutations}

The EGFR T790M mutational status was determined by ARMS (amplification refractory mutation system) with the ADx-ARMS kit (AmoyDx, Xiamen, China). EGFR mutations in plasma ctDNA were detected by using the plasma EGFR detection kit on qPCR platform. All experiments and genotype calling were performed according to the manufacturer's instructions (22).

\section{Next generation sequencing}

Next generation sequencing was performed as previously described $(19,23)$. Briefly, DNA was fragmented into an average size of $300 \mathrm{bp}$ (cfDNA without fragmentation), and $100 \mathrm{ng}$ of fragmented genomic DNA (or $10 \mathrm{ng}$ for cfDNA) was used for preparation of sequencing libraries (NEBnext Ultra II). Eight bp barcoded sequencing adaptors were then ligated to the DNA fragments and the DNA templates were amplified by PCR. Purified sequencing libraries were massively parallel sequenced by Illumina HiSeq Xten platform. 4G sequencing raw data per sample were filtered and aligned to the human reference genome.

\section{Gene-level copy number analyses}

Chromosome copy number aberrations (CNAs) were determined with the Ultrasensitive Chromosomal Aneuploidy Detector (UCAD) pipeline. Sequencing coverage for each $200 \mathrm{~K}$ bin was calculated followed by GC normalization. The sequencing coverage were further normalized by controls samples. The $\mathrm{Z}$-score for each bin was calculated by formula,

$$
Z=\frac{C_{\text {test }}-\text { mean }\left(C_{\text {control }}\right)}{\operatorname{stdev}\left(C_{\text {control }}\right)}
$$

where $C_{\text {test }}$ and $C_{\text {control }}$ are the coverage of the bin. The normalized bin values were sent to segmentation calls by algorithm circular segmentation algorithm as provided by $\mathrm{R}$ package DNAcopy. Samples with standard deviation of copy ratios between the adjacent bins $>30$ for genome-wide results were considered as with poor-quality sequence data, and these samples were excluded from this study.

\section{Statistical analysis}

R package 'DNACopy' was used for analysis of copy number changes. A P value of $<0.05$ was considered as statistically significant binary segmentation. Absolute segment value is used for further analysis. The sensitivity and specificity of UCAD were estimated by ROC curves. The chi-square test was used for categorical variables. OS was calculated from the time of development of TKI resistance to the time of death of any reason or last followup. Survival curves were constructed using the KaplanMeier method and compared using the log-rank test. All statistical analyses were performed using SPSS 17.0.

\section{Results}

\section{Patient characterization}

In this study, thirty-one enrolled lung cancer patients were collected from a previous study (clinical trial NCT02418234) which aimed to analyze the association of clinical mode and plasma T790M mutation in the relapsed patients after treatment with first generation TKI (24). Of them, 18 patients were with PFS less than 6 months and thus the tumors were considered to be with primary resistance to EGFR-TKI, 13 patients were with PFS longer than 36 months and were defined with 
Table 1 Clinicopathological information

\begin{tabular}{lcc}
\hline Clinical factors & Intrinsic resistance, $\mathrm{N}=18$ & $\begin{array}{c}\text { Acquired resistance, } \\
\mathrm{N}=13\end{array}$ \\
\hline Gender & 9 & 6 \\
Male & 9 & 7 \\
Female & & \\
EGFR baseline & 7 & 4 \\
L858R & 9 & 9 \\
19del & 1 & 0 \\
19del+L858R & 1 & 0 \\
G719X & & \\
Drug types & 9 & 9 \\
Gefitinib & 9 & 1 \\
Icotinib & 0 & 3 \\
Erlotinib & 57 & 67 \\
Age (years) & 11 & 5 \\
$\leq 60$ & 7 & \\
$>60$ & & \\
\hline
\end{tabular}

acquired EGFR-TKI resistances. As shown in Table 1, plasma T790M mutation is negatively detected in all patients by using Amplification Refractory Mutation System PCR. No statistical differences were observed for gender and EGFR baseline mutations between the two groups with primary and acquired EGFR-TKI resistance. Of note, clinical trial NCT02418234 involved 307 patients, and only these thirty-one plasma T790M negative patients shown here were found the PFS less than 6 months or more than 36 months, and our data showed that patients with primary resistance tend to be 10 -year younger than that with acquired resistance. With 60 -year as a cutoff value, we did not find statistical significance for the incidence of patients between these two groups.

\section{Cell-free DNA whole genome copy number profiling}

In this study, all raw sequencing reads were mapped to human reference genome hg19, and genomic coverage was counted by using software samtools mpileup. With these setting, we counted the average coverage for each 200k bin, and determined the significant genomic breakpoints with using circular binary segmentation algorithm. Our results showed that in all 31-tumor specimen, when compared to normal control, chromosomal breakpoints were commonly detected on centromere regions and chromosomal arm coverage imbalances were found on chromosome 1, 7 and 8 by visual inspections. In addition, chromosome 1 short arm (1p) was found with coverage lower than normalized average (as calculated as 0 ), indicating $1 \mathrm{p}$ copy loss, and chromosome 1 long arm (1q) was found with coverage higher than the normalized average, indicating 1q copy number gains. Similarly, chromosome 7 short-arm was found with higher coverage comparing to long arm, indicating chromosomal arm copy gains. Analysis further revealed a statistically significant focal amplification on chromosome $7 \mathrm{p} 11.2$, a loci where EGFR located. We also noted a copy number gain peak around $7 \mathrm{q} 31.2$. Of interest, oncogene MET, a well-known cancer driver, is located in this loci (Figure 1). In these analyses, the statistical significance of copy gain/loss was calculated by Student $t$-test by comparing chr1p 200-bin coverage values against that of health controls.

Comprehensive analysis further demonstrated statistically significant chromosome arm level changes between tumors with intrinsic resistance and with acquired resistance. Figure 2 shows a heatmap illustrating the most significant genomic changes detected. These include genetic events of $7 \mathrm{p}$ gains ( $Z \geq 3$ in $8 / 18,44.4 \%), 1 q$ heavy gains $(Z \geq 6$ in $5 / 18$, $27.8 \%), 7 \mathrm{q} 31.2$ gains $(Z \geq 3$ in $2 / 18,11.1 \%)$ and $7 \mathrm{p} 11.2$ heavy gains ( $Z \geq 6$ in $5 / 18,27.8 \%)$, frequent chromosome losses of chr18 (7/18, 38.9\%), chr9 (6/18, 33.3\%), chr3 (6/18, 33.3\%), 16p (1/18, 5.56\%), 1p (7/18, 38.9\%), chr20 (10/18, 55.6\%), 22q (4/18, 22.2\%), 10q (8/18, 44.4\%), 8p $(3 / 18,16.7 \%)$ and $\operatorname{chr} 15(6 / 18,33.3 \%)$ and chromosomal gains of $7 \mathrm{q}(5 / 18,27.8 \%), 7 \mathrm{p}(8 / 18,44.4 \%), 8 \mathrm{q}(4 / 18$, $22.2 \%)$ and $1 \mathrm{q}(6 / 18,33.3 \%)$ that are exclusively found in the group with intrinsic resistance of EGFR-TKI. On the other hand, genetic events with copy loss in chr18 (4/13, 30.8\%), chr9 (1/13, 7.69\%), chr3 (4/13, 30.8\%), $16 \mathrm{p}(2 / 13,15.4 \%), 1 \mathrm{p}(3 / 13,23.1 \%), \operatorname{chr} 20(6 / 13,46.1 \%)$, 22q (1/13, 7.69\%), 10q (6/13, 46.1\%), 8p (1/13, 7.69\%) and $\operatorname{chr} 15(2 / 13,15.4 \%)$, and genetic events with copy gain in $7 \mathrm{q}(3 / 13,23.1 \%), 8 \mathrm{q}(1 / 13,7.69 \%)$ and $1 \mathrm{q}(1 / 13$, $7.69 \%)$ were detected in the specimen from patient with lung cancer that are T790M negative but with acquired resistance to EGFR-TKI.

In addition, non-arm level copy number gains in $7 \mathrm{p} 11.2$, $22 \mathrm{q} 11.2,16 \mathrm{p} 11.2$ and $5 \mathrm{q} 31.3$, including focal amplifications, were detected in specimen from patients with lung cancer that were defined as with intrinsic resistance of EGFR-TKI with frequencies of $66.7 \%$ (12/18), $22.2 \%$ (4/18), $33.3 \%$ 


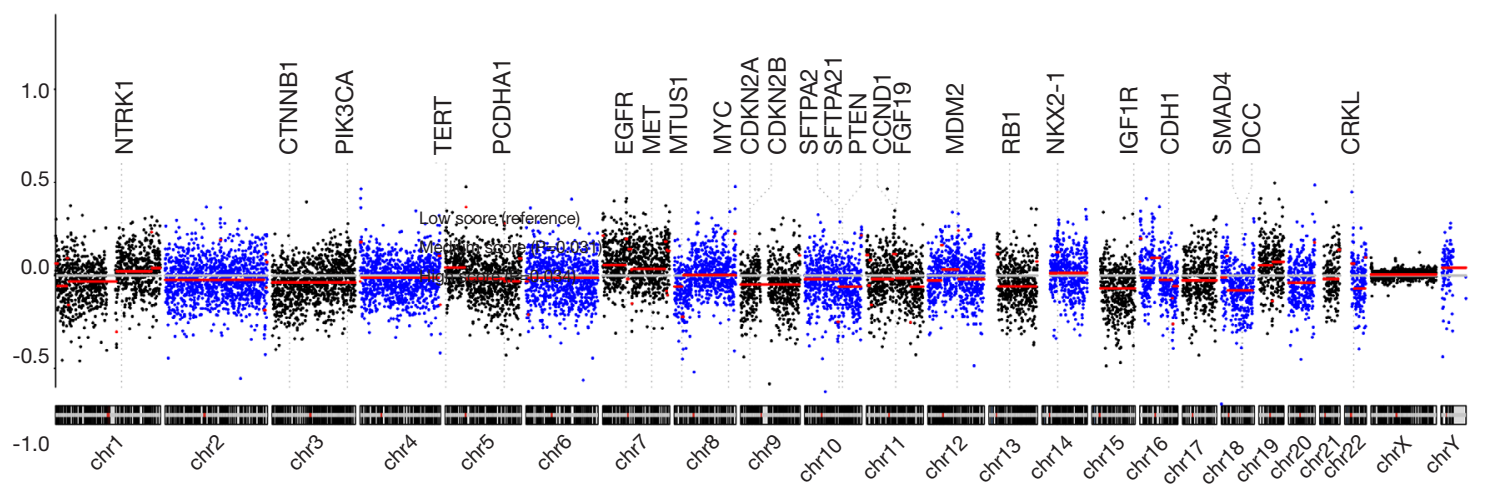

Figure 1 Plasma cell free DNA somatic copy numbers of relapsed lung cancer patients. Chromosome 1 to 22 is layout from left to right with green and black colors. Chromosomal segments are marked in red lines. Representative genes for the segments of interest are marked on the top.

T790M-, Intrinsic resitances

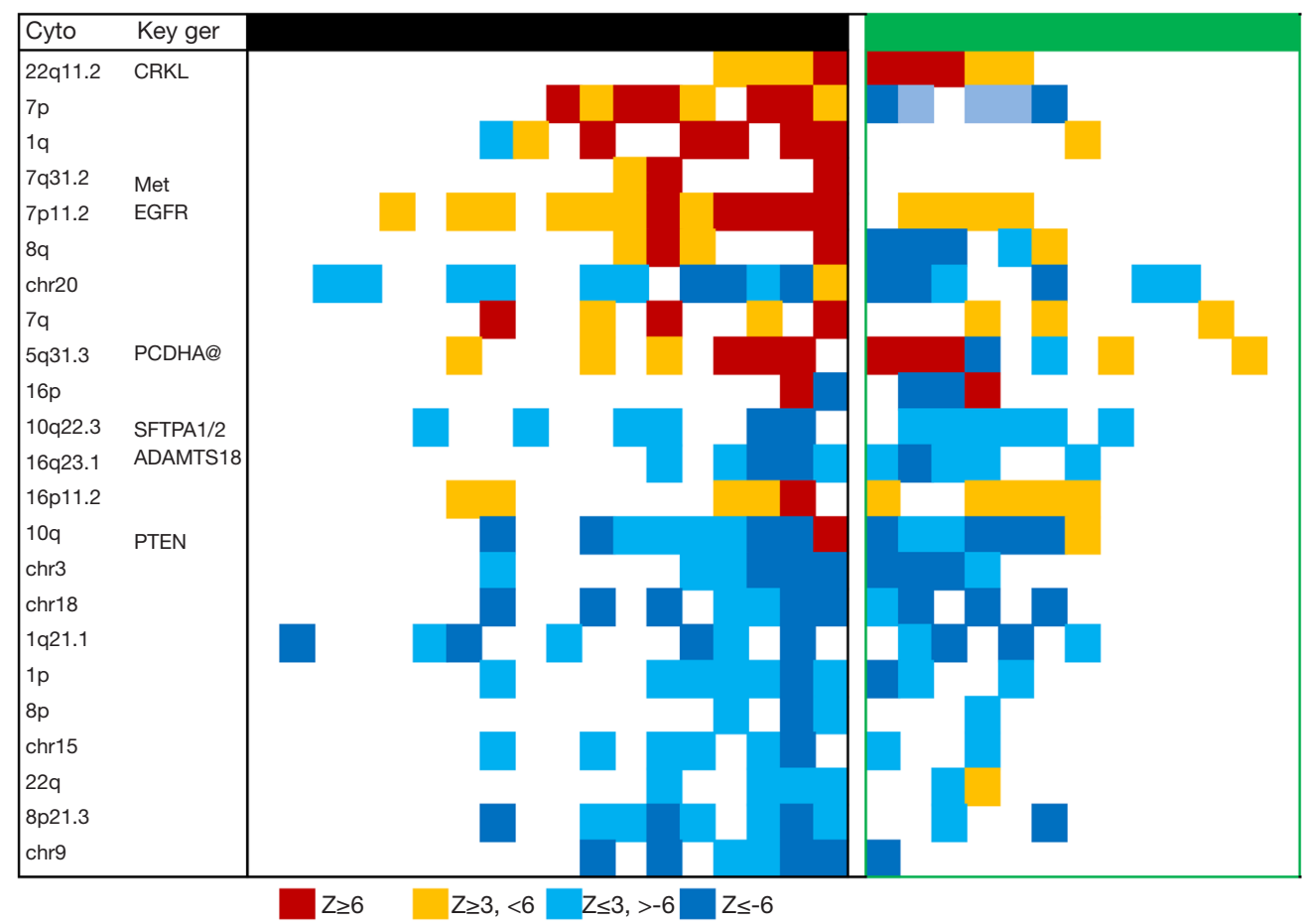

Figure 2 Heatmap view of chromosomal changes between intrinsic and acquire patients. Heatmap view of segment copy number changes in T790M negative intrinsic resistance and acquired resistance, with each line represent a segment and each column represent a sample. Significant copy-gain segments with Z-score larger than 6 are marked in red color, Z-score between 3 and 6 in orange. Significant copyloss segments with Z-score less than -6 are marked in dark blue, Z-score between -3 and -6 in light blue. The rest data points with Z-score between -3 and 3 are in white. 
Table 2 Statistical analyses of somatic copy number changes in drug resistance groups

\begin{tabular}{lccc}
\hline & \multicolumn{2}{c}{ T790M- } & \\
\cline { 2 - 3 } CNV & $\begin{array}{c}\text { Intrinsic } \\
\text { resistant, N=18 }\end{array}$ & $\begin{array}{c}\text { Acquired } \\
\text { resistant, N=13 }\end{array}$ & P value \\
\hline 7p gain & 8 & 0 & 0.009 \\
$\begin{array}{l}\text { 7p11.2 (EGFR) } \\
\text { gain }\end{array}$ & 12 & 4 & 0.073 \\
7q31.2 (MET) gain & 3 & 0 & 0.245 \\
1q gain & 6 & 1 & 0.191 \\
\hline
\end{tabular}

All the other events in Figure 2 were not listed here because of no significance.

$(6 / 18)$ and $33.3 \%(6 / 18)$, and in patients with lung cancer that were with acquired resistance with frequencies of $30.8 \%(4 / 13), 38.5 \%(5 / 13), 53.8 \%(7 / 13)$ and $38.5 \%(5 / 13)$, respectively (Figure 2). Of interest, all these amplified locations are linked with potential lung cancer oncogenes. For example, lung cancer oncogene EGFR locates in 7p11.2 (1); CRKL, a recently identified lung cancer driver predicting the relapse of patient after TKI treatment (25), locates in 22q11.2; ADAMTS18 which contributes to lung cancer development (26) locates in 16p11.2. PCDHA, methylation of this gene has been reported to be involved in multiple caner development (27), locates in 5q31.3.

\section{chr7p gains is a frequent event detected in patients with lung cancer that are EGFR T790M negative but with intrinsic TKI resistance}

We next examined the potential correlations of chromosomal copy number changes with TKI resistances. Of interest, our data showed that chromosome 7 copy number gain significantly correlates with intrinsic TKI resistances: 7p11.2 (EGFR loci) gains was detected in $66.7 \%(12 / 18)$ of lung cancer patients with intrinsic TKI resistance while it is found in only $30.7 \%$ (4/13) of patients with acquired TKI resistance; $7 \mathrm{p}$ arm gains were found in $44.4 \%(8 / 18)$ of patients with intrinsic resistance, and no such event was detected in patients with acquired TKI resistance (Fisher exact test, $\mathrm{P}=0.009$ ). However, no statistical significance was found for the other frequent detected genomic events as listed in Table 2, including $8 \mathrm{q}$ gain, $8 \mathrm{p} 21.3$ loss and $1 \mathrm{q}$ gain. The operating characteristic (ROC) analysis further revealed that chr7p copy number gains of chr7p can serve as a predictor for distinguishing

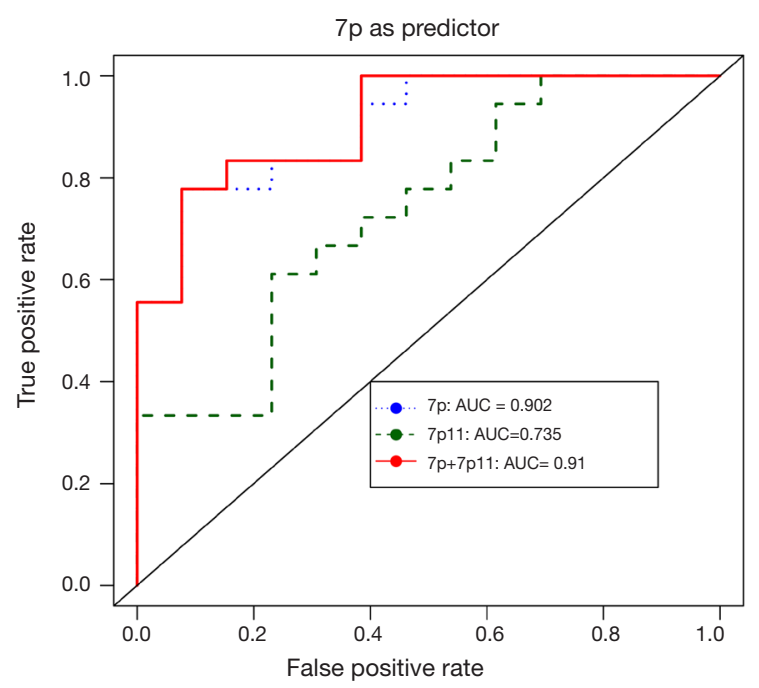

Figure 3 EGFR copy number as a predictor for EGFR-TKI intrinsic resistance in T790M negative patients. AUC curves for $7 \mathrm{p}$ gains to predict intrinsic resistance. The performance of $7 \mathrm{p} 11$ is indicated in green dash line. The performance of $7 p$ is indicated in blue dot line. The performance of combining $7 \mathrm{p}$ and $7 \mathrm{p} 11$ is indicated in red line. The combining product was estimated by $10.7 \times 7 \mathrm{p}+0.619 \times 7 \mathrm{p} 11$. The coefficients were estimated by logistical regression Category logit $(7 p+7 p 11)$, where Category is the category label of a sample, which is either 'intrinsic resistance' or 'acquired resistance'.

intrinsic resistance from acquired resistance for lung cancer in response to EGFR-TKI (Figure 3).

\section{Copy number changes correlate with overall survival (OS) after TKI resistances}

We also determined the potential correlations between these observed chromosome changes and OS of patient (Figure 4). For this, patients were grouped with short OS ( $\leq 6$ months), medium OS (6-12 months) and long OS ( $\geq 12$ months). We found that chr9 loss and 1q gain significantly correlated with shorter OS (trend tests, $\mathrm{P}=0.020$ and 0.029 respectively). In particular, patients with $1 \mathrm{q}$ gains detected had shorter OS (medium average of OS for 3.4 months) when compared to patients with 1q silent of lung cancer (medium average of OS for 22.2 months, hazard ratio $=10.97, \log$ rank test $\mathrm{P}=0.029)$. The similar association of chromosomal changes with poor OS were also observed with chr9 loss (hazard ratio $=6.48, \mathrm{P}=0.020$ ), and $7 \mathrm{q} 31.2$ gain (hazard ratio $=1.37, \mathrm{P}=0.031$ ) where oncogenes 


\begin{tabular}{|c|c|c|c|}
\hline SEGMENT & CNV & KEY GENE(S) & HR \\
\hline $1 q$ & gain & & 10.974 \\
\hline chr9 & loss & & 6.482 \\
\hline $8 q$ & gain & & 5.066 \\
\hline $7 q$ & gain & & 3.984 \\
\hline chr3 & loss & & 3.291 \\
\hline $1 p$ & loss & & 2.641 \\
\hline chr15 & loss & & 2.133 \\
\hline $8 p$ & loss & & 2.01 \\
\hline 1 cenL & loss & & 1.723 \\
\hline $\operatorname{chr} 18$ & loss & & 1.605 \\
\hline $16 p$ & gain & & 1.541 \\
\hline 2qter & loss & & 1.477 \\
\hline $7 p$ & gain & & 1.454 \\
\hline $10 q$ & loss & PTEN & 1.414 \\
\hline $7 q 31.2$ & gain & MET & 1.367 \\
\hline 16qter & loss & & 1.301 \\
\hline $7 p 11.2$ & gain & EGFR & 1.296 \\
\hline $16 p 11.2$ & gain & & 1.261 \\
\hline 5 pter & gain & & 1.238 \\
\hline $8 p 21.3$ & loss & MTUS1 & 1.18 \\
\hline $22 q$ & loss & & 1.178 \\
\hline $16 q 23.1$ & loss & ADAMTS18 & 1.142 \\
\hline 1cenR & loss & & 1.131 \\
\hline 4 pter & gain & & 1.12 \\
\hline 13 cen & gain & & 1.096 \\
\hline 10qter & gain & & 1.073 \\
\hline $22 q 11.2$ & gain & CRKL & 1.031 \\
\hline $1 q 21.1$ & loss & & 1.03 \\
\hline $5 q 31.3$ & gain & PCDHA@ & 1.003 \\
\hline $10 q 22.3$ & loss & SFTPA $1 / 2$ & 0.99 \\
\hline 18qter & gain & & 0.938 \\
\hline 6qter & gain & & 0.724 \\
\hline 5qter & loss & & 0.562 \\
\hline chr20 & loss & & 0.148 \\
\hline
\end{tabular}

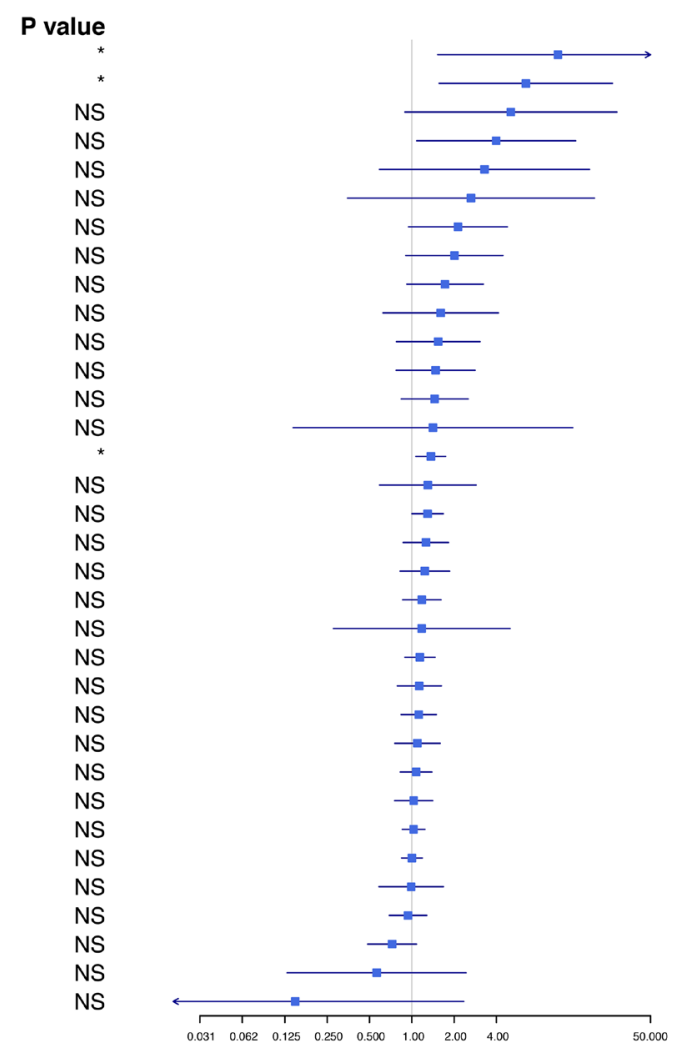

Figure 4 Genetical events which predicts overall survival. Forest plots for odd ratios of predicting intrinsic resistances for each chromosomal segment. The odd ratios are estimated by logistical regression Category logit (segment_of_interest), where Category is the category label of a sample, which is either 'intrinsic resistance' or 'acquired resistance'. Odd ratios are sorted and plot from top to bottom. Statistically significant segments (P value $<0.05)$ are marked with stars $\left(^{*}\right)$.

CDKN2A/B and MET locate $(28,29)$. No such correlations were found for other genetic events such as $8 \mathrm{p}$ loss, $1 \mathrm{p}$ loss, $8 \mathrm{p} 21.3$ loss, 7q gain, 8q gain and 16p11.2 loss, however.

\section{Discussion}

EGFR-targeted therapy has shown superior efficacy for patients with EGFR mutation. However, about 20\% to $30 \%$ patients with advanced stage lung cancers that have EGFR activating mutation show intrinsic resistance to TKI. Understanding the mechanism of the intrinsic EGFR-TKI resistance is thus important for helping improve clinical practice for lung cancer.

In this study, we identified multiple somatic copy number variations $(\mathrm{CNV})$ of chromosome through plasma cfDNA sequencing. These variants include chromosome arm level changes, focal amplifications and deletions. In mammalian cells, chromosome mis-segregation can result in chromosomal arm level changes, which affects the gene structure of many tumor genes, such as PTEN deletions on chr10 and SMAD4 deletions on chr18 (30,31). Focal amplifications, or focal chromosomal CNAs, have been discovered in cancer as critical genetic events of cancer driver gene activation resulting from many selection events during the evolution of cancer genomes (32). Our results showed that chromosomal breakpoints on centromere regions and $\mathrm{CNV}$ of chromosomes on Chromosome 1 (1p copy loss and 1q copy gain) and chromosome 7 (7p11.2 focal amplification and $7 \mathrm{q} 31.2$ copy gain) are frequently detected in plasma cfDNA of patients that were resistant to EGFR-TKI (as with either intrinsic or acquired resistance). Other chromosomal genetic variations detected frequently in the plasma cfDNA of these patients include chromosome losses of chr19, chr9, chr3, chr20, chr15, 16p, 22q, 10q and $8 \mathrm{p}$, and chromosomal gains of $8 \mathrm{q}$. Of interest, data analysis further revealed the certain patterns of chromosomal 
somatic $\mathrm{CNV}$ that may correlate to the intrinsic or acquired resistance to EGFR-TKI treatment in lung cancer patients. For example, $7 \mathrm{p}$ gains detected in the plasma cfDNA achieve highest correlation with predictable intrinsic EGFR-TKI resistance (ROC $=0.93$ ).

As regarding these chromosomal genetic variations, it is of interest that many lung cancer driver genes or tumor suppressor genes locate in the chromosome locus that have detectable changes in patients with lung cancer that are resistant to EGFR-TKI, as shown in this study. Of them, PCDHA gene cluster are the most commonly hyper-methylated genes discovered in human cancers $(26,27)$. CRKL and EGFR are two well-studied lung cancer oncogenes: CRKL amplification was discovered as one of the acquired resistances to kinase inhibitors in lung cancers treated with EGFR inhibitors (25); EGFR amplification is one of the most common genetic events in lung cancer (33), and gain of EGFR amplifications has been proposed as one of the potential drug resistance mechanism of EGFR tyrosine kinase inhibitors $(34,35)$. In our research, EGFR (7p11.2) copy gains were detected in 16/31 (51.6\%) plasma samples. CRKL (22q11.2) copy gains were detected in $8 / 31$ (25.8\%) plasma samples. Of note, however, CRKL and EGFR copy gains were detected in separate samples (Table S1), it is thus suggested that these two genetic events, if they are corresponding to, are independent to EGFR-TKI resistance. On the other hand, our study also suggest that EGFR gene amplification can drive EGFR-TKI resistance, with gene amplification may results in a ligand independent kinase domain activation leading to intrinsic TKI resistance while secondary EGFR mutation is a result of genetic selection for gained resistance during TKI treatment.

TKI resistance remains as a clinical challenge for lung cancer management. In this study, we determined the chromosomal somatic copy number changes in plasma samples of patients with TKI resistance by using clinical achievable pipeline ultra-sensitive chromosomal aneuploid detector (UCAD). Our results not only identified the most frequently detectable chromosomal somatic copy number variants that are associated with TKI resistance, but also revealed patterns that may specifically correlate with either intrinsic or acquired EGFR-TKI resistance. Our data also demonstrated that some of the chromosomal somatic copy number variants such as $7 \mathrm{p}$ gain and $1 \mathrm{q}$ gain predict worse survival of the patients. These novel findings have significant clinic impacts for guiding lung cancer treatment. For examples, EGFR gene amplification (7p gain) in lung cancer cells may result in intrinsic resistance to TKI, and these patients may benefit from clinical management with addition of cetuximab, an anti-EGFR monoclonal antibody that target EGFR amplification; Chr1q gain is another frequent genetic event identified in lung cancer patients with intrinsic resistance to TKI, and 1q21.3-encoded S100 calcium-binding protein (S100A) family members and IL-1 receptor-associated kinase 1 (IRAK1) which can be targeted by a small-molecule kinase inhibitor, pacritinib; The examination of the potential $7 \mathrm{p}$ gains and $1 \mathrm{q}$ gain in the plasma samples of lung cancer patients with UCAD may provide a useful tool for monitoring EGFR-TKI response and drug resistance assessment in patient.

\section{Conclusions}

In this study, we identified multiple somatic CNV in distinguishing EGFR-TKI intrinsic and acquired resistance through plasma cfDNA sequencing. The results were from a small-scale prospective study, including 31 cancer patients and 10 health controls. The data present here uncovered encouraging findings for mechanism and biomarkers for EGFR-TKI resistance. However, a large prospective clinical trial to further confirm these discoveries is urgently needed.

\section{Acknowledgments}

Funding: This work was supported by grants from Projects of Science and Technology Project of Hangzhou Bureau (20170533B28), the Zhejiang Provincial Natural Science Foundation (LY19H160032 and LQ20H160020) and National Natural Science Foundation of China (81602671, 81602555 and 81773242), Major project of Hangzhou Science and Technology Bureau (20180417A01).

\section{Footnote}

Conflicts of Interest: The authors have no conflicts of interest to declare.

Ethical Statement: The authors are accountable for all aspects of the work in ensuring that questions related to the accuracy or integrity of any part of the work are appropriately investigated and resolved. The protocol was approved by the Institutional Review Board of Hangzhou First People's Hospital (No. HZFH2015-47-01). All patients signed the informed consent.

Open Access Statement: This is an Open Access article 
distributed in accordance with the Creative Commons Attribution-NonCommercial-NoDerivs 4.0 International License (CC BY-NC-ND 4.0), which permits the noncommercial replication and distribution of the article with the strict proviso that no changes or edits are made and the original work is properly cited (including links to both the formal publication through the relevant DOI and the license). See: https://creativecommons.org/licenses/by-nc-nd/4.0/.

\section{References}

1. Lynch TJ, Bell DW, Sordella R, et al. Activating mutations in the epidermal growth factor receptor underlying responsiveness of non-small-cell lung cancer to gefitinib. N Engl J Med 2004;350:2129-39.

2. Reck M, Rabe KF. Precision Diagnosis and Treatment for Advanced Non-Small-Cell Lung Cancer. N Engl J Med 2017;377:849-61.

3. Herbst RS, Heymach JV, Lippman SM. Lung cancer. N Engl J Med 2008;359:1367-80.

4. Rotow J, Bivona TG. Understanding and targeting resistance mechanisms in NSCLC. Nat Rev Cancer 2017;17:637-58.

5. Pao W, Miller VA, Politi KA, et al. Acquired resistance of lung adenocarcinomas to gefitinib or erlotinib is associated with a second mutation in the EGFR kinase domain. PLoS Med 2005;2:e73.

6. Mok TS, Wu YL, Ahn MJ, et al. Osimertinib or PlatinumPemetrexed in EGFR T790M-Positive Lung Cancer. N Engl J Med 2017;376:629-40.

7. Gou LY, Li AN, Yang JJ, et al. The coexistence of MET over-expression and an EGFR T790M mutation is related to acquired resistance to EGFR tyrosine kinase inhibitors in advanced non-small cell lung cancer. Oncotarget 2016;7:51311-9.

8. Carrera S, Buque A, Azkona E, et al. Epidermal growth factor receptor tyrosine-kinase inhibitor treatment resistance in non-small cell lung cancer: biological basis and therapeutic strategies. Clin Transl Oncol 2014;16:339-50.

9. Sequist LV, Soria JC, Goldman JW, et al. Rociletinib in EGFR-mutated non-small-cell lung cancer. $\mathrm{N}$ Engl J Med 2015;372:1700-9.

10. Abbosh C, Birkbak NJ, Wilson GA, et al. Phylogenetic ctDNA analysis depicts early-stage lung cancer evolution. Nature 2017;545:446-51.

11. Kuznetsova AY, Seget K, Moeller GK, et al. Chromosomal instability, tolerance of mitotic errors and multidrug resistance are promoted by tetraploidization in human cells. Cell Cycle 2015;14:2810-20.

12. Jamal-Hanjani $M$, Wilson GA, McGranahan N, et al. Tracking the Evolution of Non-Small-Cell Lung Cancer. N Engl J Med 2017;376:2109-21.

13. Bakhoum SF, Landau DA. Chromosomal Instability as a Driver of Tumor Heterogeneity and Evolution. Cold Spring Harb Perspect Med 2017. doi: 10.1101/cshperspect. a029611.

14. Beroukhim R, Mermel CH, Porter D, et al. The landscape of somatic copy-number alteration across human cancers. Nature 2010;463:899-905.

15. Zojer N, Dekan G, Ackermann J, et al. Aneuploidy of chromosome 7 can be detected in invasive lung cancer and associated premalignant lesions of the lung by fluorescence in situ hybridisation. Lung Cancer 2000;28:225-35.

16. Ricke RM, van Deursen JM. Aneuploidy in health, disease, and aging. J Cell Biol 2013;201:11-21.

17. Norton ME, Jacobsson B, Swamy GK, et al. Cell-free DNA analysis for noninvasive examination of trisomy. $\mathrm{N}$ Engl J Med 2015;372:1589-97.

18. Stover DG, Parsons HA, Ha G, et al. Association of CellFree DNA Tumor Fraction and Somatic Copy Number Alterations With Survival in Metastatic Triple-Negative Breast Cancer. J Clin Oncol 2018;36:543-53.

19. Jiang $\mathrm{P}$, Chan $\mathrm{CW}$, Chan $\mathrm{KC}$, et al. Lengthening and shortening of plasma DNA in hepatocellular carcinoma patients. Proc Natl Acad Sci U S A 2015;112:E1317-25.

20. Xia S, Huang CC, Le M, et al. Genomic variations in plasma cell free DNA differentiate early stage lung cancers from normal controls. Lung Cancer 2015;90:78-84.

21. Jackman D, Pao W, Riely GJ, et al. Clinical definition of acquired resistance to epidermal growth factor receptor tyrosine kinase inhibitors in non-small-cell lung cancer. J Clin Oncol 2010;28:357-60.

22. Liu X, Lu Y, Zhu G, et al. The diagnostic accuracy of pleural effusion and plasma samples versus tumour tissue for detection of EGFR mutation in patients with advanced non-small cell lung cancer: comparison of methodologies. J Clin Pathol 2013;66:1065-9.

23. Liang D, Lv W, Wang H, et al. Non-invasive prenatal testing of fetal whole chromosome aneuploidy by massively parallel sequencing. Prenat Diagn 2013;33:409-15.

24. Zhang S, Zhu L, Xia B, et al. Epidermal growth factor receptor (EGFR) T790M mutation identified in plasma indicates failure sites and predicts clinical prognosis in non-small cell lung cancer progression during firstgeneration tyrosine kinase inhibitor therapy: a prospective 
observational study. Cancer Commun (Lond) 2018;38:28.

25. Suda K, Mizuuchi H, Murakami I, et al. CRKL

amplification is rare as a mechanism for acquired resistance to kinase inhibitors in lung cancers with epidermal growth factor receptor mutation. Lung Cancer 2014;85:147-51.

26. Jin H, Wang X, Ying J, et al. Epigenetic identification of ADAMTS18 as a novel 16q23.1 tumor suppressor frequently silenced in esophageal, nasopharyngeal and multiple other carcinomas. Oncogene 2007;26:7490-8.

27. El Hajj N, Dittrich M, Haaf T. Epigenetic dysregulation of protocadherins in human disease. Semin Cell Dev Biol 2017;69:172-82.

28. Caballero OL, Cohen D, Liu Q, et al. Loss of chromosome arms $3 p$ and $9 p$ and inactivation of P16 (INK4a) in normal epithelium of patients with primary lung cancer. Genes Chromosomes Cancer 2001;32:119-25.

29. Glukhova L, Lavialle C, Fauvet D, et al. Mapping of the $7 \mathrm{q} 31$ subregion common to the small chromosome 7 derivatives from two sporadic papillary renal cell carcinomas: increased copy number and overexpression of the MET proto-oncogene. Oncogene 2000;19:754-61.

30. Fujisawa H, Reis RM, Nakamura M, et al. Loss of heterozygosity on chromosome 10 is more extensive in

Cite this article as: Zhu L, Liang J, Xia B, Xu Y, Qian Z, Ma $\mathrm{S}$, Zhang S. Identification of somatic copy number variations in plasma cell free DNA correlating with intrinsic resistances to EGFR targeted therapy in T790M negative non-small cell lung cancer. J Thorac Dis 2020;12(3):883-892. doi: 10.21037/ jtd.2019.12.97 primary (de novo) than in secondary glioblastomas. Lab Invest 2000;80:65-72.

31. Blaker H, von Herbay A, Penzel R, et al. Genetics of adenocarcinomas of the small intestine: frequent deletions at chromosome $18 \mathrm{q}$ and mutations of the SMAD4 gene. Oncogene 2002;21:158-64.

32. Krijgsman O, Carvalho B, Meijer GA, et al. Focal chromosomal copy number aberrations in cancerNeedles in a genome haystack. Biochim Biophys Acta 2014;1843:2698-704.

33. Levva S, Kotoula V, Kostopoulos I, et al. Prognostic Evaluation of Epidermal Growth Factor Receptor (EGFR) Genotype and Phenotype Parameters in Triplenegative Breast Cancers. Cancer Genomics Proteomics 2017;14:181-95.

34. Tokumo M, Toyooka S, Ichihara S, et al. Double mutation and gene copy number of EGFR in gefitinib refractory non-small-cell lung cancer. Lung Cancer 2006;53:117-21.

35. Nukaga S, Yasuda H, Tsuchihara K, et al. Amplification of EGFR Wild-Type Alleles in Non-Small Cell Lung Cancer Cells Confers Acquired Resistance to MutationSelective EGFR Tyrosine Kinase Inhibitors. Cancer Res 2017;77:2078-89. 
Table S1 CRKL (22q11.2) copy gains, EGFR (7p11.2) copy gains and 7p gains in T790M primary and acquired resistance samples

\begin{tabular}{|c|c|c|c|c|c|}
\hline \multirow{2}{*}{ Molecular group } & \multirow{2}{*}{ Patient group } & \multirow{2}{*}{ ID } & \multicolumn{3}{|c|}{ Z-scores } \\
\hline & & & $22 q 11.2$ & $7 p$ & $7 p 11.2$ \\
\hline & T790M- AR & AD15085 & 8.13 & -6.27 & 1.41 \\
\hline \multirow[t]{9}{*}{ 22q11.2 positive, $7 p$ positive } & T790M-AR & AD15077 & 7.74 & -5.81 & 4.71 \\
\hline & T790M- AR & ST016 & 3.92 & -5.26 & 3.30 \\
\hline & T790M- AR & AD15054 & 3.08 & -3.08 & 5.10 \\
\hline & T790M- AR & AD15055 & 8.27 & 1.94 & 5.00 \\
\hline & T790M- PR & ST118 & 7.35 & 4.13 & 10.89 \\
\hline & T790M- PR & ST017 & 4.55 & 6.57 & 6.32 \\
\hline & T790M- PR & AD15132 & 3.49 & 6.02 & 7.79 \\
\hline & T790M- PR & AD15124 & 4.29 & 1.70 & 7.90 \\
\hline & T790M- PR & AD15062 & 0.10 & 41.39 & 17.30 \\
\hline \multirow[t]{7}{*}{$22 q 11.2$ negative, $7 p$ positive } & T790M- PR & AD15145 & -0.89 & 2.93 & 5.92 \\
\hline & T790M- PR & ST074 & 0.51 & 5.91 & 4.19 \\
\hline & T790M- PR & AD15065 & 2.15 & 2.33 & 3.86 \\
\hline & T790M- PR & AD15162 & 0.99 & 1.61 & 3.60 \\
\hline & T790M- PR & ST041 & 0.46 & -0.64 & 3.44 \\
\hline & T790M- PR & E15073B & 0.10 & 4.36 & 3.40 \\
\hline & T790M- PR & AD15063 & 2.25 & 6.92 & 3.20 \\
\hline \multirow[t]{26}{*}{$22 q 11.2$ negative, $7 p$ negative } & T790M- PR & AD15029 & 1.19 & 2.59 & 1.46 \\
\hline & T790M- PR & E15114B & 1.53 & 1.61 & 0.63 \\
\hline & T790M- PR & AD15076 & 1.39 & 1.56 & 1.12 \\
\hline & T790M- PR & AD15138 & 0.91 & 1.34 & 2.35 \\
\hline & T790M- PR & ST004 & 0.48 & 0.05 & 0.81 \\
\hline & T790M- PR & AD15163 & 0.38 & -0.29 & 1.12 \\
\hline & T790M- AR & ST100 & 0.48 & 1.45 & 1.50 \\
\hline & T790M- AR & AD15146 & 0.26 & 1.41 & -0.57 \\
\hline & T790M- AR & ST121 & 0.16 & 0.71 & 2.81 \\
\hline & T790M- AR & AD15060 & 1.52 & 0.46 & 0.38 \\
\hline & T790M- AR & ST040 & 0.95 & -0.34 & 1.12 \\
\hline & T790M- AR & ST038 & -0.14 & -1.13 & 0.78 \\
\hline & T790M-AR & AD15091 & -1.40 & -1.49 & -0.02 \\
\hline & T790M- AR & AD15061 & -0.82 & -8.83 & -1.28 \\
\hline & HEALTH_CTRL & PG10 & 1.17 & 1.42 & 0.66 \\
\hline & HEALTH_CTRL & PG09 & 0.54 & 1.13 & 0.06 \\
\hline & HEALTH_CTRL & PG07 & 0.36 & 0.71 & -1.21 \\
\hline & HEALTH_CTRL & PG13 & 0.27 & 0.60 & 1.77 \\
\hline & HEALTH_CTRL & PG04_L3 & -1.97 & 0.51 & -0.69 \\
\hline & HEALTH_CTRL & PG11 & 0.37 & 0.48 & 1.00 \\
\hline & HEALTH_CTRL & PG15 & -0.01 & 0.22 & -1.30 \\
\hline & HEALTH_CTRL & PG08 & 0.18 & -0.44 & -1.15 \\
\hline & HEALTH_CTRL & PG12 & 0.50 & -0.66 & 0.07 \\
\hline & HEALTH_CTRL & PG06_L2 & 1.10 & -0.84 & 1.12 \\
\hline & HEALTH_CTRL & PG14 & -0.76 & -1.31 & -0.45 \\
\hline & HEALTH_CTRL & PG05_L3 & -1.75 & -1.81 & 0.13 \\
\hline
\end{tabular}

$A R$, acquired resistance; PR, primary resistance. 